\title{
The Age-Dependent Contribution of Aortic Incident and Reflected Pressure Waves to Central Blood Pressure in African-Americans
}

\author{
Haroon Kamran, Jason M. Lazar, Rinkesh Patel, Ilir Maraj, \\ Heather Berman, and Louis Salciccioli \\ Division of Cardiovascular Medicine, State University of New York Downstate Medical Center, Brooklyn, NY 11203-2098, USA \\ Correspondence should be addressed to Jason M. Lazar, jason.lazar@downstate.edu
}

Received 2 March 2011; Revised 24 June 2011; Accepted 28 June 2011

Academic Editor: Samy I. McFarlane

Copyright (c) 2011 Haroon Kamran et al. This is an open access article distributed under the Creative Commons Attribution License, which permits unrestricted use, distribution, and reproduction in any medium, provided the original work is properly cited.

Aging is associated with increased central aortic systolic pressure (CSP) and pulse pressure which are predictive of cardiovascular events. Mechanisms implicated for higher central pressures include a higher forward incident pressure wave (P1), higher augmented pressure (AP), and shorter reflected wave round trip travel time (Tr). African-Americans (AA) have more frequent and deleterious blood pressure elevation. Using applanation tonometry, we studied the association of age and CSP with P1 and AP in 900 AA subjects. Data showed that in subjects $\leq 50$ years old, CSP was mediated by AP but not P1 or Tr, whereas in those $>50$, CSP was mediated by both AP and P1 and to a lesser extent by Tr. Predictive models were significant $\left(R^{2}=0.97\right)$ for both age groups. In conclusion, wave reflection is the primary determinant of CSP in younger AA, while in older subjects, CSP is mediated by both the magnitude and timing of wave reflection as well as aortic impedance.

\section{Introduction}

Vascular aging and remodeling predominantly affects the large elastic arteries, with the unfavorable consequence of increased aortic stiffness and higher central systolic (CSP) and pulse pressure (PP) [1]. In recent years, it has been demonstrated that central pressures are more closely related to cardiovascular outcomes as compared to peripheral pressures [2]. Pathological changes within the arterial system lead to hemodynamic alterations that are reflected in the aortic waveform. The growing use of applanation tonometry has rejuvenated interest in the mechanisms of blood pressure elevation. Several mechanisms have been proposed for the higher central pressures observed with aging. There may be an increased forward incident pressure wave (P1), a higher augmented pressure (AP) and augmentation index (AI), and a shorter round trip travel time of the reflected wave (Tr) [3]. Aortic characteristic impedance also increases with age, thereby increasing P1. Greater AP due to increased wave reflection is the conventional explanation of why CSP increases with age, with age-related increases in aortic stiffness shortening $\operatorname{Tr}$ and causing the reflected wave to sum on the incident wave during systole [4-8]. Other investigators have found $\mathrm{P} 1$ to be the more important determinant of central pressure $[9,10]$. A recent study in a large cohort of normal subjects found the contribution of AP and P1 to the CSP to vary between those younger and older than 60 years [11]. Thus, the reasons for higher CSP and PP with advancing age have not been fully clarified.

African-Americans (AA) have a high prevalence of hypertension, and greater propensity for end-organ damage including left ventricular hypertrophy [12]. They have also been reported to have higher aortic stiffness [13]. The agerelated contributions of the incident and reflected waves to CSP in this group of individuals have not been well studied. Accordingly, we studied the age-related contribution of the incident and reflected waves to CSP and PP in a large cohort of AA subjects with and without cardiovascular disease.

For our analysis, we divided the cohort into 2 age groups, $\leq 50$ years and those $>50$ years old. This is based on prior 
studies demonstrating AI to increase until age 60 and then level off, and that aortic stiffness measured by pulse wave velocity $(\mathrm{PWV})$ increases predominantly in those $>50$ years old $[14,15]$. Additionally, in this current study, we found that age correlated with $\mathrm{P} 1$ only in those subjects $>50$ years old.

\section{Materials and Methods}

The hospital institutional review board approved this study, and all patients provided written consent. We prospectively studied 900 AA subjects (age $58 \pm 17$ years) without known congestive heart failure. Inclusion criteria were age $\geq 18$ years, adequate radial and carotid pulses to obtain the applanation tonometry study, and sinus rhythm. Peripheral systolic (S) and diastolic (D) blood pressures (BP) were measured using an automated sphygmomanometer device (Omron HEM-780). Pulse pressure (PP) was defined as the difference between SBP and DBP. Brachial artery mean arterial pressure (MAP) was calculated as diastolic pressure $+1 / 3$ PP. The central aortic waveform was derived from the radial artery waveform with the use of applanation tonometry (Sphygmocor, Atcor Medical) and a validated transfer function $[16,17]$. The reflected wave pressure (AP) amplitude was defined as the difference between peak SBP and pressure at the inflection point of the aortic waveform [18]. The AI was defined as the proportional increase in SBP due to the reflected wave and was expressed as a percentage of the PP, and since heart rate dependent was also corrected for heart rate of $75 \mathrm{bpm}$ (AI75) [18]. Tr is defined as the time from the initial upstroke of the pressure wave to the incident pressure P1. P1 is defined as the pressure difference between the inflection point and the diastolic pressure. The reflected wave systolic duration $(\Delta \mathrm{Tr})$ is determined from the inflection point to the incisura [18]. The ejection duration is the time from the initial pressure upstroke to the incisura. These were obtained from the derived aortic waveform as previously described [18].

Clinical characteristics evaluated included age, gender, medication use, body mass index, history of hyperlipidemia, diabetes mellitus, hypertension, chronic renal insufficiency, known coronary artery disease defined as any degree of coronary disease as per patient history or chart review, previous stroke, and smoking status (current cigarette use). Hyperlipidemia, hypertension, chronic renal insufficiency, and diabetes mellitus were defined either as self-reported, documented diagnosis obtained from chart review, or current treatment with medication. As noted previously, for the statistical analysis of the age-related contributions of the waveform characteristics to central pressures, we divided our cohort into those $\leq 50$ years and those $>50$ years old.

\section{Statistics}

Data is presented as mean \pm standard deviation (SD). Differences between younger ( $\leq 50$ years) and older subjects ( $>50$ years) were assessed using independent sample $T$ test for continuous variables and with Fischer Exact or Chisquare test for dichotomous variables. The contribution of age with and without the mediation of AP and P1 to the CSBP and CPP was assessed using both mediation analysis and linear regression adjusted for the potential cofounders of gender, height, weight, HR, MAP, and CV risk factors or known coronary disease using SPSS statistical analysis software (version 17). Mediation analysis was performed both with and without the inclusion of medication classes (beta-blocker agents, angiotensin converting enzymes inhibitors and angiotensin receptor blocking agents (ACE/ARB), and calcium channel blockers) to exclude any medication interaction. Mediation analysis was used on the premise that mediation exists when a predictor affects a dependent variable indirectly through at least 1 intervening variable, or mediator. With increasing age, there is increased $\mathrm{AP}$ and P1, and, due to these changes, CSP and PP rise; thus, the central pressure and age relationship may be mediated by AP and P1. Mediation analysis has been modified by Preacher and Hayes to include multiple mediators and covariates [19].

\section{Results}

Table 1 shows characteristics of the study subjects. Thirtytwo percent of subjects were $\leq 50$ years. Thirty seven percent were taking a beta-blocking agent, 34\% an ACE/ARB, and $18 \%$ a calcium-blocking agent. On univariate analysis, there were significant correlations between $\operatorname{AP}(r=0.55, P<.001)$ and P1 $(r=0.46, P<.001)$ with CSP before and after 50 years of age $(r=0.67, P<.001$ and $0.69, P<.001$, resp.). On adjusted multivariate linear regression, in subjects $\leq 50$, age correlated with AP $(\mathrm{B}=0.071, P=0.03)$ but not with $\mathrm{P} 1$ $(\mathrm{B}=0.02, P=0.78)$ or $\operatorname{Tr}(\mathrm{B}=-0.17, P=0.13)$. After age 50 , age correlated positively with both $\mathrm{AP}$ and $\mathrm{P} 1(\mathrm{~B}=0.19$, $P<0.001$ and $\mathrm{B}=0.36, P<0.001$, resp.) and inversely with $\operatorname{Tr}(\mathrm{B}=-0.17, P=0.007)$. Mediation analysis demonstrated in younger subjects that CSP was mediated by $\mathrm{AP}(\mathrm{B}=0.07$, $95 \% \mathrm{CI}=0.01-0.14, P<0.05)$, but not by $\mathrm{P} 1$ or $\operatorname{Tr}(\mathrm{B}=$ $-0.001,95 \% \mathrm{CI}=-0.036-0.027, P=\mathrm{NS}$ and $\mathrm{B}=-0.008$, $95 \%$ CI $=-0.03-0.0001, P=\mathrm{NS}$, resp.). In subjects $>50$, the CSP was similarly mediated by AP and P1 $(\mathrm{B}=0.16,95 \%$ $\mathrm{CI}=0.11-0.25, P<0.05$ and $\mathrm{B}=0.17,95 \% \mathrm{CI}=0.09-0.22$, $P<0.05$, resp.), and to a lesser extent by $\operatorname{Tr}(\mathrm{B}=-0.01,95 \%$ $\mathrm{CI}=-0.03--0.002, P<0.05)$. Mediation analysis adjusted models were highly significant with an $R^{2}=0.97$ in both younger and older subjects $(P<0.001$ for both). When CPP was substituted for CSP, results were similar to those for CSP. In subjects $\leq 50$ years of age, CPP was mediated only by AP $(\mathrm{B}=0.09,95 \% \mathrm{CI}=0.02-0.19, P<0.05)$ and in those age $>50$ the relationship of age with CPP was similarly mediated by $\mathrm{AP}$ and $\mathrm{P} 1(\mathrm{~B}=0.20,95 \% \mathrm{CI}=0.15-0.34$ and $\mathrm{B}=0.29$, $95 \% \mathrm{CI}=0.15-0.38, P<0.05$, resp.). Figure 1 shows changes in AP and P1 by deciles of age.

Among the significant mediators, the yearly contribution of AP to the CSP before age 50 was a standardized coefficient of $0.07 /$ year. In those above 50 years old, AP and P1 contribute similarly, with factors of 0.16 /year and $0.17 /$ year, respectively, suggesting that $\mathrm{AP}$ and $\mathrm{P} 1$ are of similar importance. The contribution of the Tr was only significant in the older group at a factor of $-0.01 /$ year. Figure 2 demonstrates 


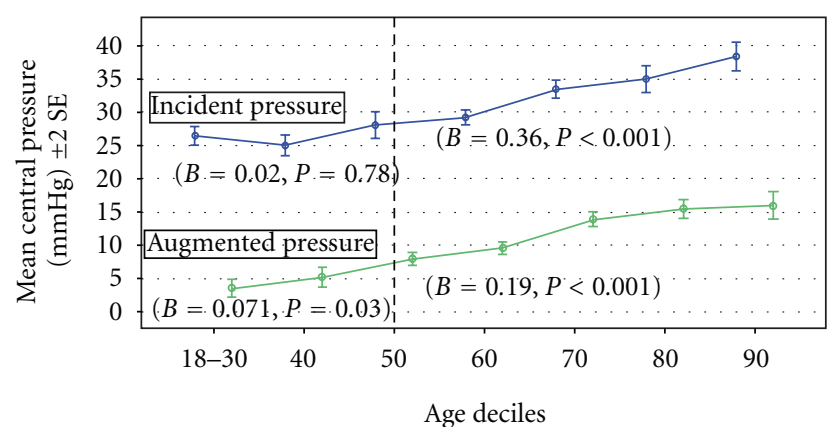

FIGURE 1: Augmented pressures and incident pressures in younger and older subjects by deciles of age.

the contribution of the mediators to CSBP in the older and younger age groups.

When analyzed by gender, among male subjects $\leq 50$, CSP was mediated by AP, but not $\mathrm{P} 1$ or $\operatorname{Tr}(\mathrm{B}=0.07,95 \%$ $\mathrm{CI}=0.03-0.15, P<0.05 ; \mathrm{B}=-0.03,95 \% \mathrm{CI}=-0.09-0.05$, $P=\mathrm{NS} ; \mathrm{B}=-0.0005,95 \% \mathrm{CI}=-0.07-0.16, P=\mathrm{NS}$, resp.). Among females $\leq 50$ years, the CSP was mediated by $\mathrm{AP}$, but not $\mathrm{P} 1$ or $\operatorname{Tr}(\mathrm{B}=0.06,95 \% \mathrm{CI}=0.20-0.14, P<0.05$; $\mathrm{B}=-0.03,95 \% \mathrm{CI}=-0.09-0.2, P<\mathrm{NS} ; \mathrm{B}=-0.008,95 \%$ $\mathrm{CI}=-0.02--0.0006, P<0.05$, resp.) (Figure 3). Among male subjects $>50$ years, CSP was mediated by AP, P1, and $\operatorname{Tr}(\mathrm{B}=0.16,95 \% \mathrm{CI}=0.09-0.30, P<0.05 ; \mathrm{B}=-0.12$, $95 \% \mathrm{CI}=0.03-0.20, P=<0.05$ and $\mathrm{B}=-0.01,95 \% \mathrm{CI}=$ $-0.0007--0.08, P \leq 0.05$, resp.). Among females $>50$ years, the CSBP was mediated by AP, $\mathrm{P} 1$ and $\operatorname{Tr}(\mathrm{B}=0.14,95 \%$ $\mathrm{CI}=0.08-0.21, P<0.05 ; \mathrm{B}=-0.20,95 \% \mathrm{CI}=0.14-0.28$, $P<$ NS; $\mathrm{B}=-0.004,95 \% \mathrm{CI}=-0.0001-0.01, P<0.05$, resp.) (Figure 3 ). When $\mathrm{CPP}$ was analyzed by gender and age group, the same mediators were found as for CSP (Figure 4).

When mediation analysis was performed with the inclusion of antihypertensive medication classes (beta blockers, $\mathrm{ACE} / \mathrm{ARB}$, and calcium channel blocking agents) as covariates, results were similar indicating that these medications had no effect on age and central pressure mediation by AP, $\mathrm{P} 1$, or Tr.

\section{Discussion}

These results suggest that in AA age $\leq 50$ the effect of age on central pressures is primarily driven by AP alone, while in those $>50$ the contribution of age towards the formation of central pressures is equally mediated by AP and P1, with a small contribution from Tr. Similar results were found for both genders. The common medication classes of bêta blockers, angiotensin-converting enzymes inhibitors and angiotensin receptor blockers, and calcium channel blockers did not substantially alter out results. The mechanism of increasing central BP with age is an area of continued interest and discussion. Higher CSP and PP are markers and potential mediators of cardiovascular disease and have been found to be predictive of cardiovascular events [20-22]. There have been opposing viewpoints with regard to the relative contributions of the incident and
TABLE 1: Clinical and hemodynamic characteristics of the study population.

\begin{tabular}{|c|c|c|c|}
\hline & $\begin{array}{l}\leq 50 \text { years } \\
(n=289)\end{array}$ & $\begin{array}{l}>50 \text { years } \\
(n=611)\end{array}$ & \\
\hline Variable & Mean \pm SD & Mean \pm SD & $P$ value \\
\hline Age (years) & $38 \pm 9$ & $67 \pm 10$ & $<0.001$ \\
\hline Males (\%) & 41 & 36 & 0.15 \\
\hline Weight (kg) & $81.8 \pm 21$ & $79 \pm 18$ & 0.07 \\
\hline Height (m) & $1.69 \pm 0.10$ & $1.67 \pm 0.10$ & 0.004 \\
\hline $\mathrm{SBP}(\mathrm{mmHg})$ & $125 \pm 21$ & $139 \pm 22.8$ & $<0.001$ \\
\hline DBP (mmHg) & $78 \pm 14$ & $79 \pm 13$ & 0.201 \\
\hline $\mathrm{PP}(\mathrm{mmHg})$ & $47 \pm 13$ & $60 \pm 19$ & $<0.001$ \\
\hline MAP (mmHg) & $93 \pm 15$ & $99 \pm 14$ & $<0.001$ \\
\hline CSBP (mmHg) & $112 \pm 20$ & $127 \pm 22$ & $<0.001$ \\
\hline $\mathrm{CDP}(\mathrm{mmHg})$ & $79 \pm 14$ & $81 \pm 12$ & 0.15 \\
\hline CPP (mmHg) & $33 \pm 11$ & $47 \pm 17$ & $<0.001$ \\
\hline CMAP (mmHg) & $90 \pm 15$ & $96 \pm 14$ & $<0.001$ \\
\hline HR (beats/min) & $74 \pm 14$ & $69 \pm 12$ & $<0.001$ \\
\hline $\mathrm{ED}(\mathrm{ms})$ & $303 \pm 37$ & $308 \pm 35$ & 0.07 \\
\hline $\mathrm{AP}(\mathrm{mmHg})$ & $6 \pm 7$ & $14 \pm 9$ & $<0.001$ \\
\hline P1 (mmHg) & $27 \pm 7$ & $33 \pm 12$ & $<0.001$ \\
\hline $\operatorname{Tr}(\mathrm{ms})$ & $144 \pm 17$ & $135 \pm 15$ & $<0.001$ \\
\hline $\operatorname{AI75}(\%)$ & $16 \pm 14$ & $25 \pm 11$ & $<0.001$ \\
\hline $\mathrm{AI}(\%)$ & $16 \pm 16$ & $28 \pm 12$ & $<0.001$ \\
\hline $\mathrm{PWV}(\mathrm{m} / \mathrm{sec})$ & $8.7 \pm 1.6$ & $9.2 \pm 1.9$ & 0.001 \\
\hline PPA & $1.45 \pm 0.2$ & $1.30 \pm 0.2$ & $<0.001$ \\
\hline $\begin{array}{l}\text { Brachial-central } \\
\text { SBP (mmHg) }\end{array}$ & $13 \pm 6$ & $12 \pm 7$ & 0.028 \\
\hline $\begin{array}{l}\text { Brachial-central } \\
\text { PP (mmHg) }\end{array}$ & $14 \pm 6$ & $12 \pm 6$ & 0.011 \\
\hline$\Delta \operatorname{Tr}(\mathrm{ms})$ & $159 \pm 37$ & $174 \pm 35$ & $<0.001$ \\
\hline HTN (\%) & 35 & 75 & $<0.001$ \\
\hline Chol (\%) & 16 & 47 & $<0.001$ \\
\hline DM (\%) & 13 & 38 & $<0.001$ \\
\hline CAD (\%) & 8 & 21 & $<0.001$ \\
\hline
\end{tabular}

Abbreviations: SBP: systolic blood pressure, DBP: diastolic blood pressure, PP: pulse pressure, MAP: mean arterial pressure, HR: heart rate, ED: ejection duration, AP: augmented pressure, P1: incident pressure, Tr: round trip travel time, AI: augmentation index, AI@75: heart rate-corrected augmentation index, PWV: pulse wave velocity, PPA: pulse pressure amplification, $\Delta \mathrm{Tr}$ : reflected wave systolic duration, HTN: hypertension, Chol: hypercholesterolemia, DM: diabetes mellitus, CAD: coronary artery disease, kg: kilograms, mmHg: millimeters of mercury, ms: milliseconds, m: meters, $\mathrm{m} / \mathrm{sec}$ : meters per second.

reflected waves to CSP and PP [3]. Greater aortic impedance may be a predominant factor, especially in older subjects $[9,23]$. The present study helps to clarify these relations among AA, a group of patients particularly vulnerable to hypertensive complications. The present study findings are similar to that of Namasivayam et al. [11], who in a healthy cohort found wave reflection to be important throughout life, while aortic impedance was significant in older subjects. They chose a partition age of 60 years for their analysis, 


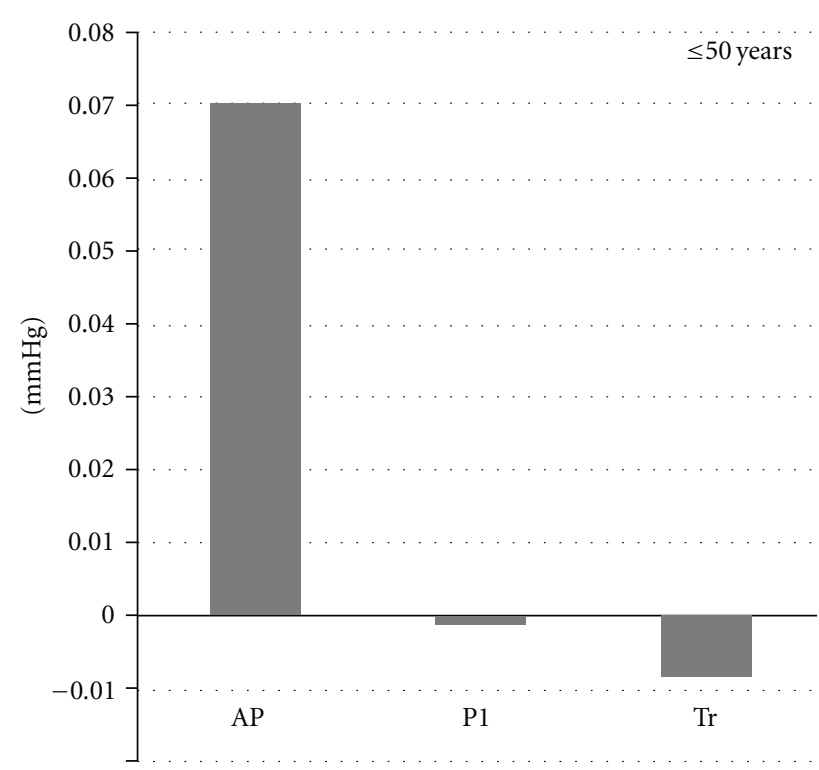

(a)

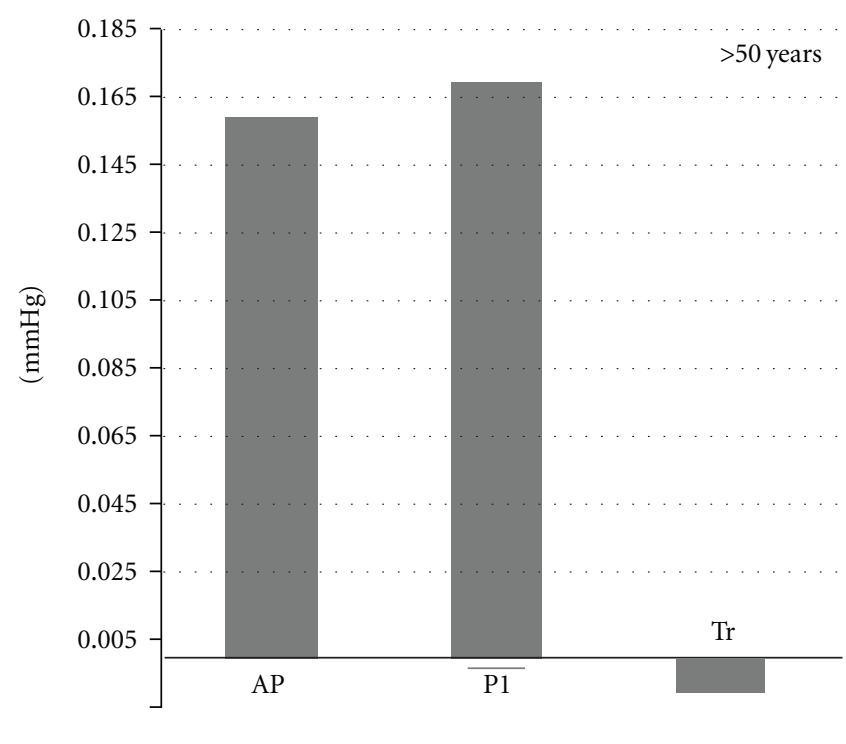

(b)

Figure 2: Per year contribution on augmented pressure (AP), incident pressure (P1), and round trip travel time younger and older subjects.

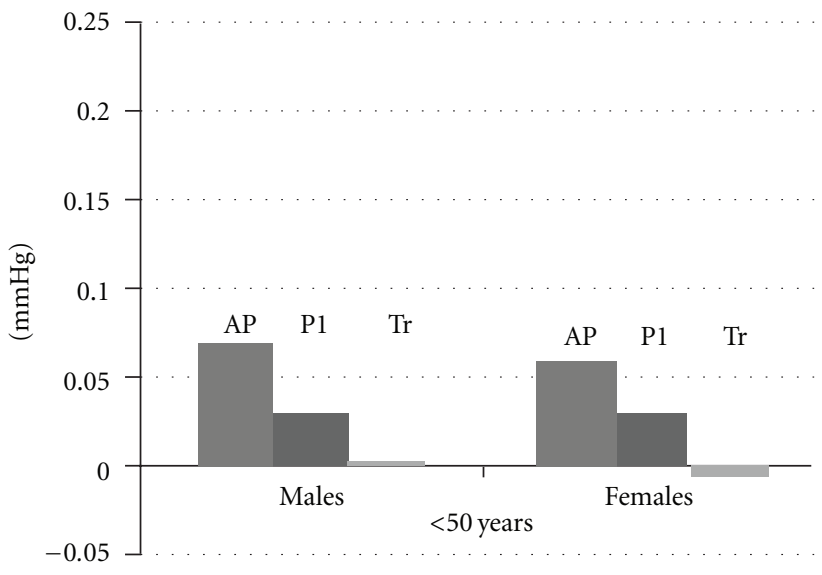

(a)

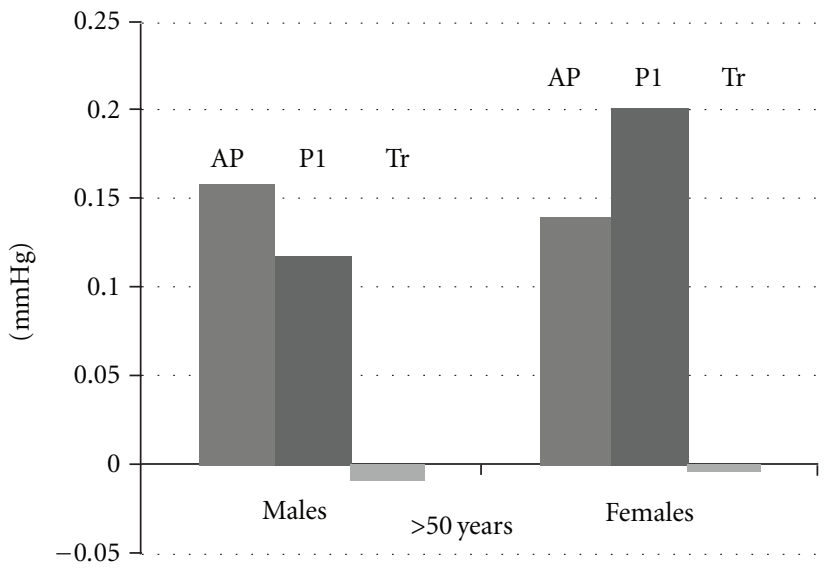

(b)

FIgURE 3: Gender-wise per year contribution of augmented pressure (AP), incident pressure (P1), and round trip travel time (Tr) to the aortic systolic pressure before and after 50 years.

based on physiologic considerations. A strength of our study is that we statistically determined 50 years to be the optimal partition based on our finding that only above this age did P1 and age correlate. This may be population dependent, since AAs have been suggested to have increased aortic stiffness as compared to whites. Regardless of the exact age, the present study underscores the facts that the reflected arterial wave continues to contribute to central aortic BP later in life. The contribution of the reflected wave was emphasized by the work of Murgo et al. [4], who found central BP augmentation related to waveform reflection and characterized waveforms in older and younger subjects. These findings have been confirmed by subsequent studies [24]. In addition, aortic stiffness and characteristic impedance may also contribute to a higher CSP, and it has been suggested that it is the primary mechanism. In a healthy middle aged to older cohort from the Framingham study, Mitchell et al. found an age-related increase in aortic PWV and the forward pressure wave, with little change or decrease in the AI [9]. Another study of hypertensive subjects found higher PP related to increased aortic wall stiffness and smaller aortic diameter even when corrected for AI [23]. Multiple studies of predominantly Caucasian subjects have shown differing changes in AI, AP, and PWV with age. AI rises in a curvilinear manner only to level off in older age although AP continues to increase throughout life. PWV remains stable in youth but increases in older subjects [15]. The curvilinear nature and plateau noted for AI is likely due to the similar 


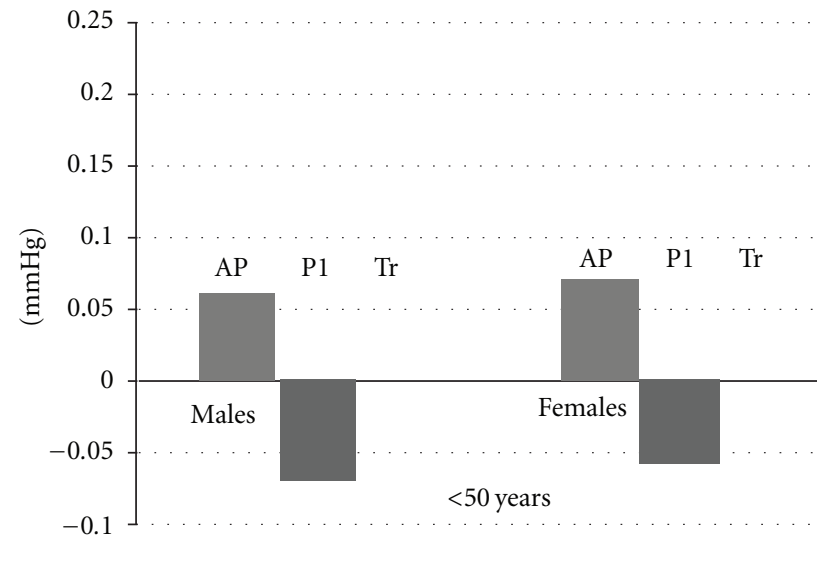

(a)

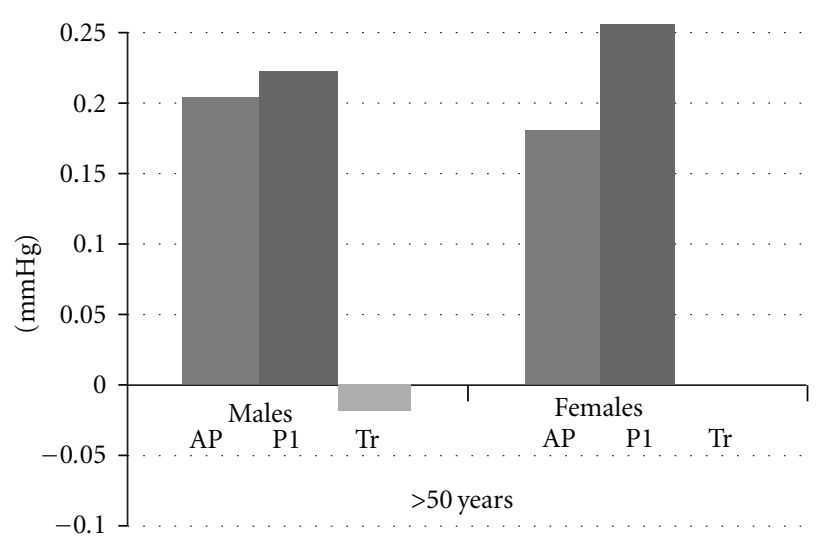

(b)

FIGURE 4: Gender-wise contribution of augmented pressure (AP), incident pressure (P1), and round trip travel time (Tr) to aortic pulse pressure before and after 50 years.

rise in both $\mathrm{AP}$ and $\mathrm{PP}$ with aging [25]. These patterns have raised concern about the utility of AI in risk stratification of older populations [9].

In younger individuals, AI rises steeply with age whereas aortic PWV does not [14]. This is consistent with the idea that the rise in aortic pressures is due to an increase in the magnitude of wave reflection rather than increased aortic stiffness. In older individuals, AI changes little, however, aortic PWV increases, suggesting that the rise in aortic pressures is driven by both the earlier return of the reflected wave and increasing aortic stiffness and not only changes in the magnitude of wave reflection alone [15]. The magnitude of reflected wave is in part dependent upon the magnitude of incident wave, and, therefore, AP continues to rise in older individuals due to increasing P1. In our study, Tr mediated CSP to a lesser extent and only in the older group.

The aorta functions as a conduit to transport blood from the heart to the periphery and as a protective cushion to lessen pulsatile flow to the end organs. With advancing age, the arteries progressively stiffen. This phenomenon is more common in the large elastic arteries compared to the more muscular peripheral arteries and results in the progressive loss of vascular elasticity, decreased cushioning effect and worsening microvascular function and end organ damage $[26,27]$. There is an increase in PP due to an increase in systolic pressure and a decrease in diastolic pressure. The two mechanisms that underlie increased PP appear to be a higher $\mathrm{P} 1$ generated by the ejection of blood from the left ventricle into a stiffened aorta, and an increase in AP due to higher P1 and a shorter Tr allowing the reflected wave to return in late systole and further augment systolic pressure [28]. Our results are consistent with these mechanisms, which result in higher central systolic and pulse pressures and are closely related to CV outcomes [2]. Left ventricular function is also adversely affected, by decreased diastolic coronary perfusion, increased left ventricular hypertrophy, and increased left ventricular workload and wasted pressure energy [29].

Our data is subject to the limitations of a cross-sectional study which restricts our ability to determine causal relationships of age and pressures. Longitudinal studies are lacking. We used patient interview and when feasible chart review to obtain clinical characteristics and history, which although standard is imperfect and may lead to error in data collection. Despite these limitations, however, our population of AA is the largest group to be studied for the agerelated waveform components of central blood pres-sure. We used both multivariate and mediation analysis controlling for covariates to help sort out these relationships.

\section{Conclusion}

In summary, this study helps to clarify the physiology of increasing central blood pressure with age. The findings in our AA cohort are similar to a previously studied mostly Caucasian normal population [11]. CSP and PP are in part mediated by wave reflection throughout life, while increasing aortic impedance is important at an older age. Treatments aimed at decreasing wave reflection, such as vasodilators, may be more efficacious in the younger hypertensive population. Therapies to lower aortic stiffness, if and when available, would likely be of greater benefit in the older age group.

\section{References}

[1] S. S. Franklin, W. Gustin, N. D. Wong et al., "Hemodynamic patterns of age-related changes in blood pressure: the Framingham Heart Study," Circulation, vol. 96, no. 1, pp. 308-315, 1997.

[2] B. Williams, P. S. Lacy, S. M. Thom et al., "Differential impact of blood pressure-lowering drugs on central aortic pressure and clinical outcomes: principal results of the Conduit Artery Function Evaluation (CAFE) study," Circulation, vol. 113, no. 9, pp. 1213-1225, 2006.

[3] R. S. Vasan, "Pathogenesis of elevated peripheral pulse pressure: some reflections and thinking forward," Hypertension, vol. 51, no. 1, pp. 33-36, 2008. 
[4] J. P. Murgo, N. Westerhof, J. P. Giolma, and S. A. Altobelli, "Aortic input impedance in normal man: relationship to pressure wave forms," Circulation, vol. 62, no. 1, pp. 105-116, 1980.

[5] A. V. Chobanian, "Isolated systolic hypertension in the elderly," The New England Journal of Medicine, vol. 357, no. 8, pp. 789-796, 2007.

[6] W. W. Nichols and M. F. O'Rourke, McDonald's Blood Flow in Arteries, Hodder Arnold, London, UK, 5th edition, 2005.

[7] S. Laurent, J. Cockcroft, L. Van Bortel et al., "Expert consensus document on arterial stiffness: methodological issues and clinical applications," European Heart Journal, vol. 27, no. 21, pp. 2588-2605, 2006.

[8] I. B. Wilkinson, H. MacCallum, P. C. Hupperetz, C. J. van Thoor, J. R. Cockcroft, and D. J. Webb, "Changes in the derived central pressure waveform and pulse pressure in response to angiotensin II and noradrenaline in man," Journal of Physiology, vol. 530, no. 3, pp. 541-550, 2001.

[9] G. F. Mitchell, H. Parise, E. J. Benjamin et al., "Changes in arterial stiffness and wave reflection with advancing age in healthy men and women: the Framingham Heart Study," Hypertension, vol. 43, no. 6, pp. 1239-1245, 2004.

[10] J. L. Izzo, "Arterial stiffness and the systolic hypertension syndrome," Current Opinion in Cardiology, vol. 19, no. 4, pp. 341352, 2004.

[11] M. Namasivayam, B. J. McDonnell, C. M. McEniery, and M. F. O'Rourke, "Does wave reflection dominate age-related change in aortic blood pressure across the human life span?" Hypertension, vol. 53, no. 6, pp. 979-985, 2009.

[12] V. E. Friedewald, S. D. Nesbitt, C. V.S. Ram, and W. C. Roberts, "Hypertension in African Americans and other non-caucasian ethnic groups," American Journal of Cardiology, vol. 106, no. 10, pp. 1466-1472, 2010.

[13] K. S. Heffernan, S. Y. Jae, K. R. Wilund, J. A. Woods, and B. Fernhall, "Racial differences in central blood pressure and vascular function in young men," American Journal of Physiology-Heart and Circulatory Physiology, vol. 295, no. 6, pp. H2380-H2387, 2008.

[14] C. M. McEniery, Yasmin, I. R. Hall, A. Qasem, I. B. Wilkinson, and J. R. Cockcroft, "Normal vascular aging: differential effects on wave reflection and aortic pulse wave velocity-the AngloCardiff Collaborative Trial (ACCT)," Journal of the American College of Cardiology, vol. 46, no. 9, pp. 1753-1760, 2005.

[15] W. Wojciechowska, J. A. Staessen, T. Nawrot et al., "Reference values in white Europeans for the arterial pulse wave recorded by means of the SphygmoCor device," Hypertension Research, vol. 29, no. 7, pp. 475-483, 2006.

[16] C. H. Chen, E. Nevo, B. Fetics et al., "Estimation of central aortic pressure waveform by mathematical transformation of radial tonometry pressure: validation of generalized transfer function," Circulation, vol. 95, no. 7, pp. 1827-1836, 1997.

[17] A. L. Pauca, M. F. O’Rourke, and N. D. Kon, "Prospective evaluation of a method for estimating ascending aortic pressure from the radial artery pressure waveform," Hypertension, vol. 38, no. 4, pp. 932-937, 2001.

[18] W. W. Nichols, "Clinical measurement of arterial stiffness obtained from noninvasive pressure waveforms," American Journal of Hypertension, vol. 18, no. 1, part 2, pp. 3S-10S, 2005.

[19] K. J. Preacher and A. F. Hayes, "Asymptotic and resampling strategies for assessing and comparing indirect effects in multiple mediator models," Behavior Research Methods, vol. 40, no. 3, pp. 879-891, 2008.
[20] J. A. Staessen, J. Gasowski, J. G. Wang et al., "Risks of untreated and treated isolated systolic hypertension in the elderly: metaanalysis of outcome trials," The Lancet, vol. 355, no. 9207, pp. 865-872, 2000.

[21] M. J. Roman, R. B. Devereux, J. R. Kizer et al., "Central pressure more strongly relates to vascular disease and outcome than does brachial pressure: the Strong Heart Study," Hypertension, vol. 50, no. 1, pp. 197-203, 2007.

[22] R. Pini, M. C. Cavallini, V. Palmieri et al., "Central but not brachial blood pressure predicts cardiovascular events in an unselected geriatric population," Journal of the American College of Cardiology, vol. 51, no. 25, pp. 2432-2439, 2008.

[23] G. F. Mitchell, P. R. Conlin, M. E. Dunlap et al., "Aortic diameter, wall stiffness, and wave reflection in systolic hypertension," Hypertension, vol. 51, no. 1, pp. 105-111, 2008.

[24] R. Kelly, C. Hayward, A. Avolio, and M. O’Rourke, "Nonivasive determination of age-related changes in the human arterial pulse," Circulation, vol. 80, no. 6, pp. 1652-1659, 1989.

[25] M. Namasivayam, A. Adji, and M. F. O’Rourke, "Aortic augmentation index and aging: mathematical resolution of a physiological dilemma?" Hypertension, vol. 56, no. 1, pp. e9e10, 2010.

[26] M. F. O'Rourke and J. Hashimoto, "Mechanical factors in arterial aging: a clinical perspective," Journal of the American College of Cardiology, vol. 50, no. 1, pp. 1-13, 2007.

[27] G. F. Mitchell, "Effects of central arterial aging on the structure and function of the peripheral vasculature: implications for end-organ damage," Journal of Applied Physiology, vol. 105, no. 5, pp. 1652-1660, 2008.

[28] G. M. London, "Large artery function and alterations in hypertension," Journal of Hypertension, Supplement, vol. 13, no. 2, pp. S35-S38, 1995.

[29] J. Hashimoto, W. W. Nichols, M. F. O’Rourke, and Y. Imai, "Association between wasted pressure effort and left ventricular hypertrophy in hypertension: influence of arterial wave reflection," American Journal of Hypertension, vol. 21, no. 3, pp. 329-333, 2008. 


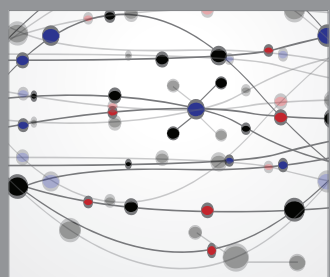

The Scientific World Journal
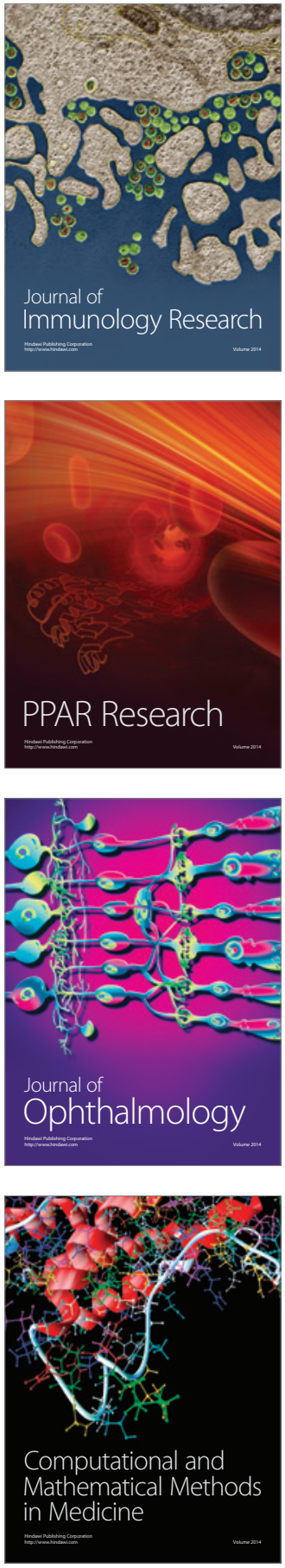

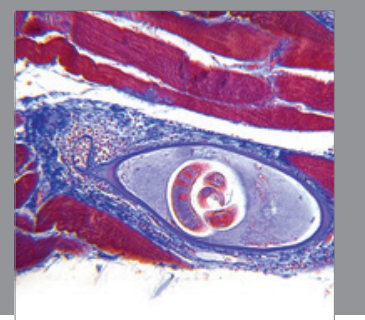

Gastroenterology

Research and Practice
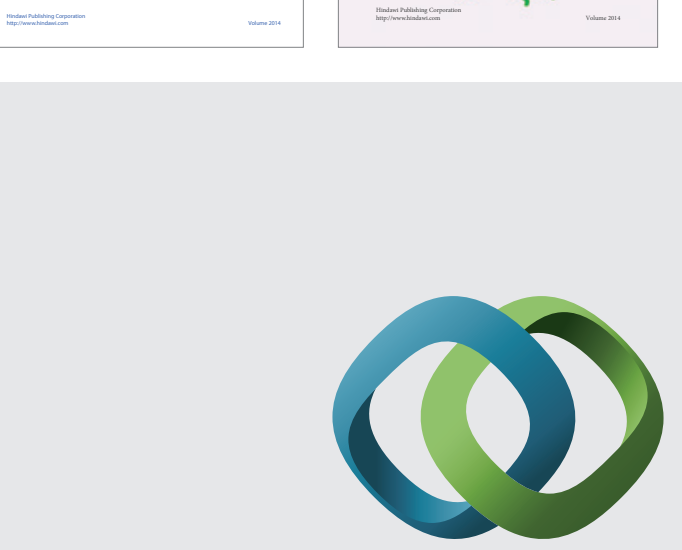

\section{Hindawi}

Submit your manuscripts at

http://www.hindawi.com
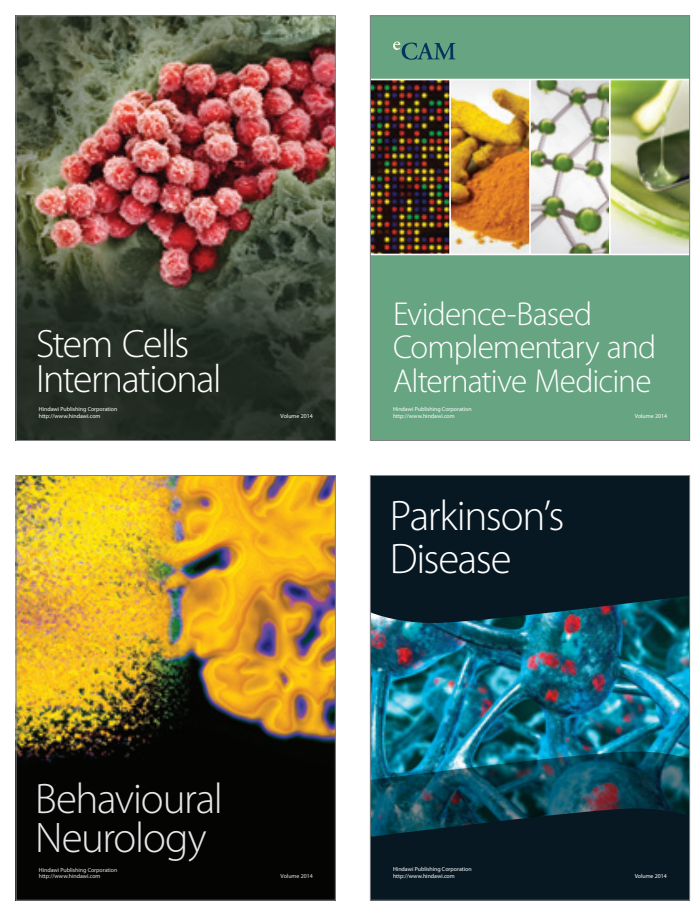

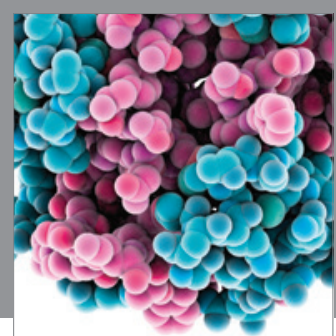

Journal of
Diabetes Research

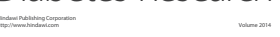

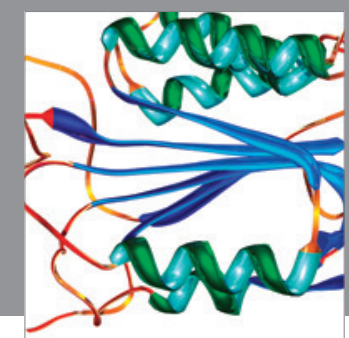

Disease Markers
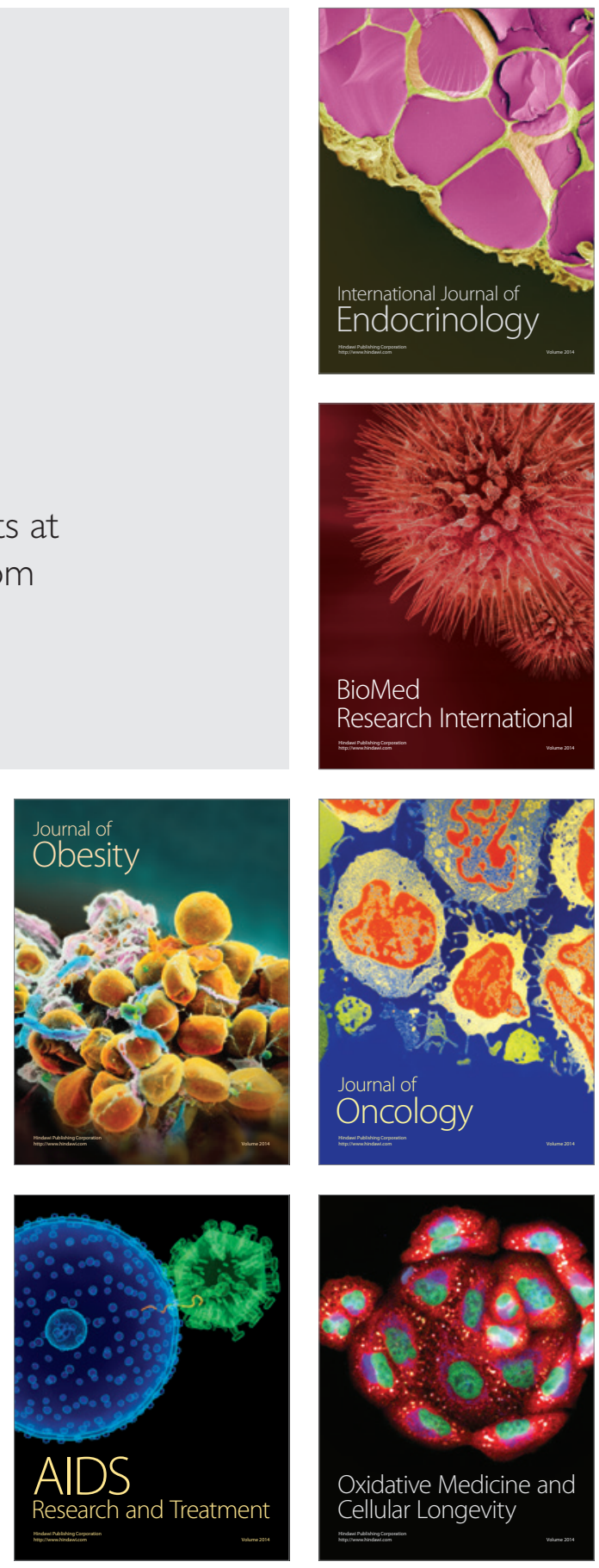